\title{
Papel da enfermagem na educação sexual de adolescentes
}

\author{
Role of nursing in sexual education of adolescents \\ Papel de la enfermería en la educación sexual de los adolescentes
}

Recebido: 10/01/2022 | Revisado: 19/01/2022 | Aceito: 27/01/2022 | Publicado: 29/01/2022

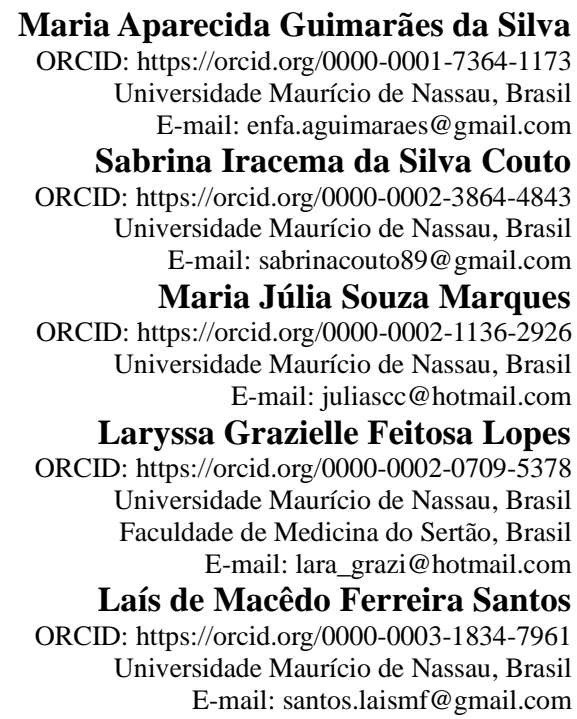

\begin{abstract}
Resumo
Objetivo: Apresentar com base na literatura científica o papel da enfermagem para a promoção da saúde sexual e o impacto na vida dos adolescentes. Metodologia: Trata-se de uma revisão integrativa da literatura, realizada nas bases de dados Literatura Latino-Americana e do Caribe em Ciências da Saúde (LILACS), Banco de Dados em Enfermagem (BDENF) e Scientific Electronic Library Online (SCIELO), foram utilizados artigos escritos na língua portuguesa, publicados no período entre 2017 e 2021. Resultados: Foram encontrados 308 artigos no total, após rigor metodológico por meio dos critérios de inclusão e exclusão, foram selecionados 14 artigos, sendo 6 artigos na LILACS, 4 na BDENF e 4 na SCIELO. A análise dos artigos selecionados foi feita de forma descritiva e predispôs a etapa de extração dos dados: autor / ano de publicação, título / base de dados e objetivo. Conclusão: De acordo com a literatura constata-se que a educação sexual é de suma importância para os adolescentes, tendo em vista que a falta dela causa impactos negativos nos aspectos biopsicossociais desse grupo. Vale ressaltar que a escola é o melhor lugar para se abordar esses adolescentes para instruí-los a respeito do autoconhecimento sobre sua opção sexual, como prevenir-se de ISTs e gravidez não planejada.
\end{abstract}

Palavras-chave: Enfermagem; Educação sexual; Adolescência.

\begin{abstract}
Objective: To present, based on scientific literature, the role of nursing in promoting sexual health and its impact on the lives of adolescents. Methodology: This is an integrative literature review, carried out in the Latin American and Caribbean Literature in Health Sciences (LILACS), Nursing Database (BDENF) and Scientific Electronic Library Online (SCIELO) databases. articles written in Portuguese, published in the period between 2017 and 2021, were used. Results: A total of 308 articles were found, after methodological rigor through the inclusion and exclusion criteria, 14 articles were selected, 6 articles in LILACS, 4 in BDENF and 4 in SCIELO. The analysis of selected articles was descriptive and predisposed to the data extraction stage: author / year of publication, title / database and objective. Conclusion: According to the literature, it appears that sex education is of paramount importance for adolescents, considering that the lack of it causes negative impacts on the biopsychosocial aspects of this group. It is noteworthy that school is the best place to approach these adolescents to educate them about self-knowledge about their sexual option, how to prevent STIs and unplanned pregnancy.
\end{abstract}

Keywords: Nursing; Sex education; Adolescence.

\section{Resumen}

Objetivo: Presentar, con base en la literatura científica, el papel de la enfermería en la promoción de la salud sexual y su impacto en la vida de los adolescentes. Metodología: Se trata de una revisión integradora de la literatura, realizada 
en las bases de datos de Literatura Latinoamericana y del Caribe en Ciencias de la Salud (LILACS), Base de Datos de Enfermería (BDENF) y Biblioteca Electrónica Científica en Línea (SCIELO). Artículos escritos en portugués, publicados en el período comprendido entre Se utilizaron 2017 y 2021 . Resultados: Se encontraron un total de 308 artículos, luego de rigor metodológico a través de los criterios de inclusión y exclusión, se seleccionaron 14 artículos, 6 artículos en LILACS, 4 en BDENF y 4 en SCIELO. El análisis de los artículos seleccionados fue descriptivo y predispuesto a la etapa de extracción de datos: autor / año de publicación, título / base de datos y objetivo. Conclusión: De acuerdo con la literatura, parece que la educación sexual es de suma importancia para los adolescentes, considerando que su falta genera impactos negativos en los aspectos biopsicosociales de este grupo. Cabe mencionar que la escuela es el mejor lugar para acercarse a estos adolescentes para educarlos sobre el autoconocimiento sobre su opción sexual, cómo prevenir las ITS y embarazos no planeados.

Palabras clave: Enfermería; Educación sexual; Adolescencia.

\section{Introdução}

A Organização Mundial da Saúde (OMS) caracteriza a adolescência como o período entre 10 a 19 anos, distinguindo, ainda, que a adolescência inicial é caracterizada pela idade de 10 anos até o final dos 14 anos, e a fase da adolescência final acontece a partir dos 15 anos e pode perdurar até os 25 anos. A adolescência é um período da vida muito importante pois é o início da aparição das características sexuais secundárias, do desenvolvimento de processos psicológicos e de padrões de identificação que evoluem da fase infantil para a adulta, através da transição de um estado para outro de relativa autonomia (câmara, 2012).

As transformações da puberdade vão além das questões biológicas e fisiológicas, essas transformações estão diretamente ligadas as relações sociais, deste modo esta fase não pode ser considerada apenas uma simples faixa etária, pois trata-se da transição para a vida adulta, portanto, é permeada por decisões sociais e principalmente psicológicas, sabendo que o adolescente está em uma constante busca para encontrar sua real personalidade, essas condições tornam essa população vulnerável pois grande parte apresenta comportamento negligente com o cuidado da saúde (Macedo, 2015; Fonseca et al., 2013).

É nesse período de transformações, que ocorre frequentemente as descobertas da sexualidade que, em um conceito mais amplo, é a energia que está contida no ser humano, independente do sexo e da idade, a qual envolve práticas e desejos interligados a diferentes formas de sentir prazer e se satisfazer. A sexualidade é a condição de ter sexo e de ser sexuado o que faz parte da natureza humana, o que insere nesse contexto a busca pelo afeto, pelo contato e pela intimidade, que se expressam na forma de sentir, no modo de se tocar e ser tocado (Lins et al., 2017; Souza et al., 2017).

Com a descoberta da sexualidade vem geralmente, a fase de início da atividade sexual. No Brasil, durante a década de 80, a média da idade para a realização da primeira relação em homens era de 15,6 anos e em mulheres 16,9 anos. Na década de 90, essa média variou para 15,3 e 16, respectivamente. A partir do ano 2000, essas idades foram menores, em que a média foi de 13,9 anos para homens e 15,5 para mulheres (Brasil, 1999; Tronco \& Dell'Aglio, 2012).

Neste período onde as questões sexuais tornam-se mais evidentes e marcantes apresenta também o início da idade reprodutiva, e os adolescentes tendem a assumir comportamentos de risco sem estarem preparados para isto, e assim, contribuir para o aumento da suscetibilidade às Infecções Sexualmente Transmissíveis (IST) ou mesmo para uma gravidez indesejada, essas questões são problemas de saúde pública visto que a sexualidade está cada vez mais precoce na vida dos adolescentes, e muitas vezes eles não possuem as informações necessárias para lidar com esta fase da vida (Queiroz, 2016; Veloso et al., 2014).

De acordo com esse cenário de vulnerabilidade se faz necessário estratégias para fornecer as informações necessárias aos adolescentes para garantir o seu conhecimento a respeito do assunto, e a escola se mostra o melhor local para alcançar esse público tendo em vista a baixa adesão dos adolescentes aos serviços de saúde. Os professores são inicialmente os imersores desse tema para os adolescentes, mas se faz necessário um trabalho em conjunto com profissionais de saúde pois estes estão 
mais preparados para lidar com a saúde sexual e reprodutiva, em especial com a sexualidade, restringindo-se ao aspecto biológico da questão (Lins et al., 2017).

Diante dessa necessidade foi criado no Brasil uma ação governamental que buscava apreender todo esse processo de integração, o Projeto Saúde e Prevenção nas Escolas (SPE). Tal projeto, lançado pelo Ministério da Saúde, foi uma iniciativa interministerial, criada em 2003 pelos Ministérios da Saúde e da Educação, cujo objetivo era a promoção da saúde sexual e reprodutiva com vistas à redução das vulnerabilidades de adolescentes e jovens: às doenças sexualmente transmissíveis (DST/HIV/Aids), à gravidez não planejada, ao uso de psicoativos. Além disso, visou fomentar o protagonismo juvenil na participação da elaboração de políticas públicas (Ribeiro \& Ribeiro, 2015).

Posteriormente no ano de 2007, o SPE foi substituído pelo Programa Saúde na Escola (PSE), que propõe uma política intersetorial entre os Ministérios da Saúde e da Educação na perspectiva da atenção integral (prevenção, promoção e atenção) à saúde de crianças, adolescentes e jovens do ensino básico público. Onde é de responsabilidade das Equipes de Saúde da Família conjuntamente com as equipes das escolas trazer eventos e consultas voltadas para esse público. Destaca-se o papel do enfermeiro junto à população escolar, apresentando desde esclarecimentos de questões fisiológicas a orientações de prevenção a IST's e gravidez na adolescência. O enfermeiro escolar deve ser parte integrante de qualquer organização educacional, visando um modelo de sistematização da assistência à saúde do escolar (Brasil, 2007; Fraenkel et al., 2008; Silva et al., 2010).

A enfermagem tem um papel fundamental na saúde dos adolescentes e no ambiente educacional diante da sua responsabilidade como promotor do conhecimento através de ações de educação em saúde, além disso o enfermeiro possui autonomia para fazer diagnósticos com base na Sistematização da Assistência de Enfermagem (SAE) da necessidade desses adolescentes, conhecendo assim a real necessidade de cada grupo através das suas indagações em relação à saúde sexual, cabe a enfermagem desenvolver estratégias para a prevenção e promoção de forma sistematizada de saúde através do diagnostico realizado (Amoras et al., 2015).

O tema da pesquisa justifica-se mediante a identificação da necessidade da implementação de diálogo sobre educação sexual com adolescentes, a importância desse diálogo foi observada através das práticas clínicas e da literatura. O presente estudo tem como objetivo principal apresentar com base na literatura científica o papel da enfermagem para a promoção da saúde sexual e o impacto na vida dos adolescentes.

\section{Metodologia}

O presente trabalho trata-se de uma Revisão Integrativa da Literatura, de caráter descritivo e abordagem quantitativa. A revisão integrativa é um método utilizado para investigar estudos já existentes com intuito de se obter resultados sobre um determinado tema, de forma ordenada e sintetizada. Para a elaboração desta revisão, foram utilizados os procedimentos metodológicos recomendados pela literatura vigente, sendo eles: 1) Identificação do tema e da questão norteadora; 2) Estabelecimento de critérios de inclusão e exclusão; 3) Categorização dos artigos; 4) Avaliação dos resultados incluídos; 5) Interpretação dos resultados; 6) Síntese do conhecimento (Souza et al., 2010; Botelho et al., 2011).

Esta pesquisa tem como questão norteadora: Qual a importância da enfermagem na educação em saúde acerca da construção do conhecimento de adolescentes sobre sexualidade?

Após rigor metodológico, as bases de dados utilizadas foram a Literatura Latino-Americana e do Caribe em Ciências da Saúde (LILACS), Banco de Dados em Enfermagem (BDENF) e a Scientific Eletronic Library OnLine (SCIELO). Para as bases de dados foram usados os descritores em Ciências da Saúde (DeCS): "Enfermagem", "Educação Sexual" e "Adolescência".

O levantamento foi realizado no mês de setembro de 2021, atendendo a alguns critérios de inclusão e exclusão. Foram excluídos trabalhos incompletos, artigos de opinião, ensaios teóricos, relatos de experiência, resumos expandidos, artigos de 
reflexão, revisões da literatura, dissertações, teses e artigos que não cumpriam o período e temática estipulados. Foram incluídos na pesquisa apenas estudos completos, sendo artigos originais, que atendam ao objetivo desta pesquisa, escritos na língua portuguesa, publicados no período entre 2017 a 2021, e em revistas nacionais ou internacionais.

\section{Resultados}

Foram encontrados 308 artigos no total, após rigor metodológico por meio critérios de inclusão e exclusão, foram selecionados 14 artigos nas bases de dados, sendo 6 na LILACS, 4 na BDENF e 4 na SCIELO. A forma de seleção descrita pode ser visualizada na Figura 1.

Figura 1: Esquema da seleção dos artigos.

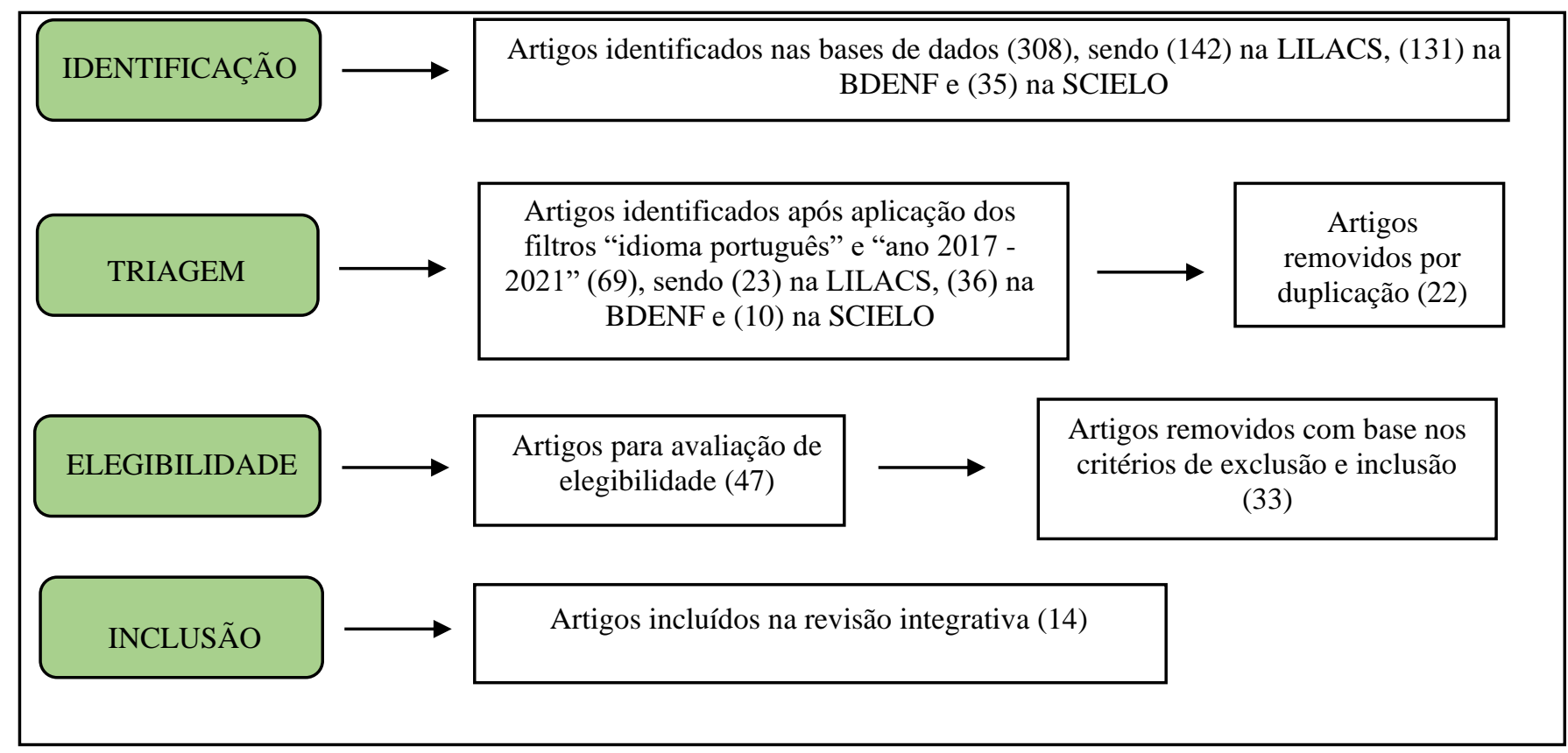

Fonte: Autores (2021).

Observou-se, durante a pesquisa nas bases de dados, que predominaram artigos com data de publicação inferior ao período estipulado nesta pesquisa, o que reforça a importância de atualizar esta temática. Bem como observou-se também que dos 14 artigos, 10 foram publicados em revistas voltadas para a Enfermagem, e os outros 4 em revistas da saúde. Após criteriosa análise dos artigos selecionados, a seleção das informações foi feita de forma descritiva e predispôs a etapa de extração dos dados (Quadro 1). 
Quadro 1: Classificação dos artigos quanto a autor / ano de publicação, título / base de dados e objetivo.

\begin{tabular}{|c|c|c|}
\hline AUTOR / ANO & TÍTULO / BASE & OBJETIVO \\
\hline Almeida et al / 2017 & $\begin{array}{l}\text { Conhecimento de adolescentes relacionados às } \\
\text { doenças sexualmente transmissíveis e gravidez / } \\
\text { SCIELO }\end{array}$ & $\begin{array}{l}\text { Investigar o conhecimento de adolescentes relacionado } \\
\text { às Infecções Sexualmente Transmissíveis (IST), AIDS } \\
\text { e gravidez, além de conhecer a compreensão sobre o } \\
\text { papel da escola na educação sexual }\end{array}$ \\
\hline da Silva et al / 2017 & $\begin{array}{l}\text { Pesquisa-ação: promovendo educação em saúde } \\
\text { com adolescentes sobre infecção sexualmente } \\
\text { transmissível / BDENF }\end{array}$ & $\begin{array}{l}\text { Identificar as dúvidas dos alunos de uma escola pública } \\
\text { federal sobre Infecção Sexualmente Transmissível e } \\
\text { propor uma abordagem ou metodologia educacional } \\
\text { mais apropriada para os alunos. }\end{array}$ \\
\hline Freire et al / 2017 & $\begin{array}{l}\text { Aspectos psicossociais da sexualidade na } \\
\text { adolescência: diálogos e aprendizagem na escola / } \\
\text { LILACS }\end{array}$ & $\begin{array}{l}\text { Compreender os aspectos psicossociais da sexualidade } \\
\text { de adolescentes de uma escola pública do município de } \\
\text { Juazeiro/BA, revelados através do desenvolvimento de } \\
\text { oficinas reflexivas }\end{array}$ \\
\hline Genz et al / 2017 & $\begin{array}{l}\text { Doenças sexualmente transmissíveis: conhecimento } \\
\text { e comportamento sexual de adolescentes / SCIELO }\end{array}$ & $\begin{array}{l}\text { Avaliar o conhecimento e comportamento sexual de } \\
\text { adolescentes sobre doenças sexualmente transmissíveis }\end{array}$ \\
\hline $\begin{array}{l}\text { Pinheiro, Silva \& } \\
\text { Tourinho / } 2017\end{array}$ & $\begin{array}{l}\text { A estratégia saúde da família e a escola na } \\
\text { educação sexual: uma perspectiva } \\
\text { intersetorialidade / SCIELO }\end{array}$ & $\begin{array}{l}\text { Analisar como o trabalho de educação sexual de } \\
\text { adolescentes e jovens é desenvolvido na perspectiva da } \\
\text { intersetorialidade entre saúde e educação }\end{array}$ \\
\hline Santos et al / 2017 & $\begin{array}{l}\text { A prevenção do vírus da imunodeficiência humana } \\
\text { pela equipe de atenção primária voltada aos } \\
\text { adolescentes / BDENF }\end{array}$ & $\begin{array}{l}\text { Investigar as ações de promoção e prevenção do HIV } \\
\text { desenvolvidas para adolescentes pela equipe de } \\
\text { enfermagem na atenção primária }\end{array}$ \\
\hline $\begin{array}{l}\text { de Andrade Ferreira et } \\
\text { al / } 2018\end{array}$ & $\begin{array}{l}\text { Adolescentes no espaço escolar e o conhecimento a } \\
\text { respeito da saúde sexual e reprodutiva / LILACS }\end{array}$ & $\begin{array}{l}\text { Analisar o conhecimento de adolescentes sobre saúde } \\
\text { sexual e reprodutiva no espaço escolar }\end{array}$ \\
\hline $\begin{array}{l}\text { Brasil, Cardoso \& } \\
\text { Silva / } 2019\end{array}$ & $\begin{array}{lccc}\begin{array}{l}\text { Conhecimento } \\
\text { sexualmente } \\
\text { contraceptivos / BDENF }\end{array} & \begin{array}{c}\text { de } \\
\text { transmissíveis }\end{array} & \begin{array}{c}\text { sobre } \\
\text { e }\end{array} & \begin{array}{c}\text { infecções } \\
\text { métodos }\end{array} \\
\end{array}$ & $\begin{array}{l}\text { Avaliar o nível de conhecimento de escolares sobre } \\
\text { Infecções Sexualmente Transmissíveis e métodos } \\
\text { contraceptivos }\end{array}$ \\
\hline Ferreira et al / 2019 & $\begin{array}{l}\text { Sexualidade na percepção de adolescentes } \\
\text { estudantes da rede pública de ensino: contribuição } \\
\text { para o cuidado / LILACS }\end{array}$ & $\begin{array}{l}\text { Discutir a percepção de adolescentes acerca da } \\
\text { sexualidade no espaço escolar }\end{array}$ \\
\hline Sehnem et al / 2019 & $\begin{array}{l}\text { Saúde sexual e reprodutiva dos adolescentes: } \\
\text { percepções dos profissionais em enfermagem / } \\
\text { LILACS }\end{array}$ & $\begin{array}{l}\text { Conhecer como é percebida e abordada a saúde sexual } \\
\text { e reprodutiva dos adolescentes pelos enfermeiros na } \\
\text { atenção primária à saúde }\end{array}$ \\
\hline Franco et al / 2020 & $\begin{array}{l}\text { Educação em saúde sexual e reprodutiva do } \\
\text { adolescente escolar / BDENF }\end{array}$ & $\begin{array}{l}\text { Relatar a experiência de estudantes do Curso de } \\
\text { Enfermagem na implementação de intervenções } \\
\text { educacionais para a promoção da saúde sexual e } \\
\text { reprodutiva do adolescente escolar }\end{array}$ \\
\hline Silva et al / 2020 & $\begin{array}{l}\text { Diagnóstico do conhecimento dos adolescentes } \\
\text { sobre sexualidade / SCIELO }\end{array}$ & $\begin{array}{l}\text { Realizar o diagnóstico do conhecimento dos } \\
\text { adolescentes sobre sexualidade para a implementação, } \\
\text { à posteriori, de um programa específico e direcionado } \\
\text { de intervenção }\end{array}$ \\
\hline Andrade et al / 2021 & $\begin{array}{l}\text { Conhecimento de adolescentes e jovens sobre } \\
\text { questões relacionadas ao sexo, em uma escola } \\
\text { pública de Monte Alegre do Piaú-PI / LILACS }\end{array}$ & $\begin{array}{l}\text { Avaliar o conhecimento de adolescentes e jovens sobre } \\
\text { questões relacionadas ao sexo, na cidade de Monte } \\
\text { Alegre do Piauí (PI) }\end{array}$ \\
\hline Vieira et al / 2021 & $\begin{array}{l}\text { Conhecimentos de adolescentes } \\
\begin{array}{l}\text { contraceptivos } \\
\text { co infecções }\end{array} \\
\text { transmissíveis / LILACS }\end{array}$ & $\begin{array}{l}\text { Identificar os conhecimentos de adolescentes sobre } \\
\text { práticas sexuais seguras e identificar as necessidades } \\
\text { de informação dos adolescentes sobre infecções } \\
\text { sexualmente transmissíveis e gravidez }\end{array}$ \\
\hline
\end{tabular}

Fonte: Autores (2021).

\section{Discussão}

\subsection{Educação em saúde sexual}

Na família, o diálogo sobre sexualidade e sexo, no geral é visto como um tabu. Segundo Andrade et al. (2021), a comunicação dos adolescente com os pais sobre assuntos relacionado a sexo é limitada quando comparada à quantidade que buscam informações através da internet, amigos e escola. A dificuldade de diálogo com os pais, é justificada por estes não saberem abordar o tema com os filhos e por acreditarem que esse tipo de conversa incentiva a prática, ou ainda, porque atribuem valor negativo ao assunto reproduzindo a mesma conduta de seus pais. Essa falta de diálogo esclarecedor com a família, provoca impactos negativos na sexualidade dos jovens, pois acabam obtendo informações não seguras, podendo desenvolver comportamentos distorcidos e vulneráveis (Freire et al., 2017). 
Os pais, em muitos casos, transferem a responsabilidade da educação sexual para a escola, pois torna-se menos complexo deixar que a escola se responsabilize em trabalhar essa temática com os adolescentes. De acordo com uma pesquisa realizada por Jones (2010), evidenciou-se que $2 / 3$ dos adolescentes entrevistados nunca falaram sobre esse assunto com os pais, logo, os professores constituem a primeira opção de fonte de conhecimento sobre sexualidade (Almeida et al., 2017; Freire et al., 2017).

Nesse contexto, a escola se torna um local privilegiado, pois é onde os adolescentes passam a maior parte do seu tempo. Porém, no âmbito escolar as práticas educacionais voltadas a sexualidade ainda se restringem as aulas de ciências biológicas, o que torna a educação uma prática realizada de forma assistemática e descontínua, com uma abordagem estritamente biológica, ignorando assim os outros aspectos psicossociais (da Silva et al., 2017; Genz et al. 2017; Ferreira et al., 2019; Sehnem et al., 2019; Franco et al., 2020).

A educação sexual na escola é considerada uma ação básica, e deve ter como finalidade capacitar os adolescentes para aquisição da autonomia e autocuidado em relação a sua sexualidade. Sendo assim tornam-se imprescindíveis práticas educativas direcionadas à sexualidade e a saúde reprodutiva dos adolescentes. Para que a elaboração dessas práticas seja eficiente é necessário considerar o nível de conhecimento desses escolares acerca do tema (da Silva et al., 2017; Silva et al. 2020; Franco et al., 2020).

A educação sexual deve reconhecer a sexualidade como construção social, pois é necessário compreender que as práticas sexuais e os desejos são também construídos culturalmente. Portanto, a orientação sexual serve para que os adolescentes possam compreender o desenvolvimento sexual, a saúde reprodutiva, as relações de gênero, as relações interpessoais, e as afetivas, a imagem corporal e autoestima. Com o intuito de reduzir comportamentos de riscos e práticas sexuais não seguras (da Silva et al., 2017; de Andrade et al., 2018).

Se faz necessário a qualificação dos professores, embora estes reconheçam a importância do tema, a maioria alega não possuir conhecimento suficiente para promover uma orientação adequada a esses adolescentes. Para Genz et al (2017), para que a educação sexual seja realizada de forma eficiente nas escolas é necessária uma intervenção interativa e divertida. Acredita-se que gincanas é uma boa forma de garantir a participação e atenção dos alunos, pois os alunos vão manter o foco e ficar atentos a cada fase das atividades desenvolvidas (Brasil et al., 2019).

\subsection{Consequências da falta de educação sexual para adolescentes}

A sexualidade na adolescência é considerada um problema de saúde pública em todo mundo, mais de dez milhões de infectados pelo HIV estão dentro da faixa etária entre 15 a 24 anos de idade. De acordo com Andrade et al. (2021), no Brasil, entre os anos de 2005 e 2015, o número de casos entre os adolescentes duplicou, demostrando um grave problema de saúde pública, com importante relevância epidemiológica. No que diz respeito as outras ISTs também podem deixar sequelas que podem ser reversíveis ou não, como infertilidade, gravidez ectópica, câncer genital, entre outros malefícios (Almeida et al., 2017; Silva et al., 2020).

Os adolescentes em sua maioria não conhecem seu próprio corpo, e mostram-se incapazes de reconhecer os sintomas das ISTs, o que deixar uma porta aberta para o contágio. Esse grupo ainda relata que não conhece nenhuma forma de contágio para infecções, isso mostra que apesar da difusão das informações pelas mídias sociais estas informações não estão chegando nos adolescentes. Essa desinformação se configura em uma situação de risco, tendo em vista que esse público fica exposto a situações que podem influenciar no número de aumento de parceiros sexuais e de relações desprotegidas, o que aumenta a chance de exposição a infecções e gravidez indesejada (Almeida et al., 2017; Genz et al., 2017; Andrade et al., 2021).

A antecipação da atividade sexual em adolescentes e a falta de informações resulta em uma maior vulnerabilidade o que leva a uma sequência de comportamentos de risco à saúde. Boa parte dos adolescentes acreditam que é impossível estar 
infectados com qualquer IST sem saber, associam o fato que uma pessoa com aparência saudável não pode estar infectada, o que aumenta as chances de contaminação e disseminação (Almeida et al., 2017; da Silva et al., 2017; Brasil et al., 2019).

Nos dias atuais ainda existe a crença generalizada que, para se contrair uma IST, é necessário ter relações sexuais com várias pessoas. Esse falso conhecimento leva esse público a menosprezar o uso do preservativo, sendo que as atividades sexuais desprotegidas trazem grandes repercussões negativas para a saúde. Andrade et al. (2021), trouxe em seu trabalho que as práticas de sexo oral e anal estão cada vez mais frequentes entre os adolescentes, e são grandes potencializadores da disseminação das ISTs nesse público por serem feitas de forma desprotegida (da Silva et al., 2017).

De acordo com Brasil et al. (2019), o baixo nível de conhecimento dos jovens ao que se refere à sexualidade, reflete o seu despreparo para iniciar a vida sexual de forma segura. É considerado assustador a quantidades de adolescentes que consideram a pílula do dia seguinte como segundo método contraceptivo mais conhecido depois do preservativo. Os adolescentes tendem a só procurar o serviço de saúde apenas quando apresentam algum sintoma, porém a maioria das ISTs são assintomáticas. Dessa forma os infectados têm um alto potencial de transmissão por não saberem que estão contaminados. (Freitas et al., 2017; Santos et al., 2017; Franco et al., 2020).

Os adolescentes precisam se empoderar em relação aos assuntos sobre sexualidade, e a como cuidar da sua vida sexual e reprodutiva, para que isso aconteça é necessário o auxílio dos serviços de saúde, junto com as instituições educacionais. É necessário incentivar que esse público procure os serviços de saúde, mesmo que no primeiro contato eles estejam envergonhados e preocupados. Cabe ao profissional de saúde encontrar estratégias para superar esses sentimentos e estabelecer um vínculo de confiança, através de uma relação respeitosa e acolhedora (Sehnem et al., 2019; Silva et al., 2020).

\subsection{O papel da enfermagem na educação sexual para adolescentes}

A educação em saúde constitui um tema que cada vez mais tem ocupado o espaço nas discussões e reflexões entre os profissionais de saúde, principalmente os que atuam em nível público na atenção básica, como os enfermeiros. A realização de um trabalho de proteção e promoção a saúde, assegurado pela Lei 8080/90, representa a abertura das portas para o autocuidado, o que é, de fato um direito do indivíduo. Essa prática tem se mostrado como uma fonte de prevenção que visa garantir a dignidade da pessoa humana. Educar para saúde é ir além da assistência curativa, é priorizar ações preventivas é reconhecer os usuários dos serviços de saúde como protagonistas no processo saúde-doença, estimulando-os a lutarem por mais qualidade da vida (Santos et al., 2017; Brasil et al., 2019).

Acredita-se, que por meio da disseminação do autocuidado, tendo o profissional de enfermagem como o principal agente, é de suma importância a conscientização dos escolares sobre orientações acerca da sexualidade. Embora o tema sexualidade na adolescência seja um tema que vem ganhando bastante abordagem na atualidade, ainda se encontram barreiras para trabalhar esse assunto em ambiente escolar. Portando, a enfermagem inserida nesse contexto apresenta um papel importa de educador na atuação das práticas educativas integradas que contemplem as necessidades desse grupo (Almeida et al., 2017; Brasil et al., 2019).

O profissional da enfermagem apresenta-se como instrumento primordial para fortalecer o trabalho de educação em saúde junto aos adolescentes, visando auxiliar os escolares a lidar com a sexualidade com responsabilidade e assim minimizar os agravos a saúde desse grupo. A enfermagem tem como objetivo ajudar as pessoas na aquisição e recuperação de habilidades, para cuidar de si. Ao se discutir à luz da prática do autocuidado a educação em saúde valoriza a responsabilidade individual e coletiva como um mecanismo no entendimento das necessidades humanas, ou seja, ele se torna uma ajuda ao aprender viver (da Silva et al, 2017; Brasil et al., 2019).

O papel do enfermeiro educador é auxiliar os adolescentes a tomar decisões conscientes baseadas em informações claras, por isso precisa desempenhar um papel de facilitador para estes, nesse sentido o profissional deve compreender o 
contexto em que os escolares estão inseridos, levando em consideração as suas situações vivenciadas, seus sentimentos e necessidades. A educação em saúde, no contexto da enfermagem, vem sendo uma realidade incontestável, e apresenta mudanças nos paradigmas da atenção à saúde. Entende-se que o ensino, junto aos adolescentes desenvolve nesse grupo a prática do autocuidado (da Silva et al, 2017; Sehnem et al., 2019).

Para que a educação em saúde aconteça no cuidado ao adolescente, o enfermeiro precisa ampliar suas intervenções na realidade de saúde, além das consultas individuais de enfermagem que contemplam principalmente as dúvidas em especiais aquelas que esse grupo tem vergonha de abordar no coletivo. A enfermagem tem como objetivo assistir os adolescentes de forma global, e pra isso se faz necessário ter outros espaços além da Unidade de Saúde da Família (USF). Nas escolas o enfermeiro pode abordar, individual ou em coletivo esses adolescentes, devendo utilizar estratégias criativas para o esclarecimento de dúvidas, e disseminação de conhecimento sobre a saúde sexual e reprodutiva (Sehnem et al., 2019).

O Programa Saúde na Escola (PSE), é uma política de atenção à saúde do adolescente no Brasil que visa a prevenção de doenças em escolares, esse programa tem parceria com as equipes de saúde da família. Porém ainda esbarra em diversas dificuldades e uma delas é a formação dos recursos humanos, tendo em vista que muitos profissionais da educação não estão capacitados, nem sensibilizados para trabalhar esse tema com os alunos, como já mencionado, muitos desses profissionais acreditam que os adolescentes não têm maturidade, nem autonomia suficiente para exercer plenamente seus direitos (Pinheiro et al., 2017; Santos et al., 2017).

Para uma efetiva funcionalidade do PSE torna-se importante o trabalho em equipe entre professores e profissionais de saúde, principalmente enfermeiros, junto aos adolescentes. A vista disso é importante que esse público seja alcançado antes mesmo do início das atividades sexuais, para assim poder ajudá-los a lidar com a sexualidade de uma forma responsável e positiva, devendo ocorrer de forma que respeite o sigilo e a privacidade do adolescente (Freire et al., 2017; Ferreira et al., 2019).

Para alcançar esse público no ambiente escolar, os profissionais de saúde, sobretudo, o enfermeiro da USF deve delinear um caminho para chegar até os adolescentes e promover junto com os profissionais de educação, orientações sobre sexualidade no geral. Segundo da Silva et al. (2017) são estratégias para o ensino sobre orientação sexual, e prevenção de IST medidas como: palestras sobre sexualidade realizadas pelos profissionais de saúde, na escola; educação permanente com os servidores, para dinamizar o diálogo sobre a sexualidade; grupo de apoio a família para incentivar o diálogo sobre sexualidade; rodas de conversas com os adolescentes sobre educação sexual e prevenção de ISTs. Só dessa forma que a educação em saúde vai favorecer uma interação do profissional com o adolescente, pois visa à aprendizagem compartilhada e à formação coletiva dos conhecimentos (Franco et al., 2020).

\section{Conclusão}

De acordo com a literatura constata-se que a educação sexual é de suma importância para os adolescentes, tendo em vista que a falta dela causa impactos negativos nos aspectos biopsicossociais desse grupo. Vale ressaltar que a escola é o melhor lugar para se abordar esses adolescentes para instruí-los a respeito do autoconhecimento sobre sua opção sexual, como prevenir-se de ISTs e gravidez não planejada.

Com a revisão da literatura foi possível observar que mesmo a escola sendo o melhor lugar para implementar a educação sexual, ainda se encontra muitas barreiras para que esse assunto seja bem difundido para os adolescentes. Um dos principais problemas encontrado é a falta de habilidade e conhecimento dos professores para instruir os alunos de forma correta sobre o autocuidado e autoconhecimento.

Através do exposto conclui-se sobre a importância do papel da enfermagem, principalmente os que estão a frente das USFs como enfermeiro educador que além do papel em ensinar os adolescentes, tem a responsabilidade de capacitar os 
professores sobre a temática. Além de ter que desenvolver estratégias para promover uma educação sexual de qualidade na escola, os profissionais de enfermagem têm a incumbência de criar uma relação de confiança com os adolescentes para que eles possam se abrir de forma que retirem todas as suas dúvidas sem medo de julgamentos e de serem expostos aos seus familiares.

Conclui-se que estratégias que trabalhem os temas sexualidade da forma lúdica e onde tenha interação do aluno na construção da atividade se mostrou mais positiva em relação do aprendizado, pois quando o aluno participa da construção do conhecimento ele se empenha para se sair bem na atividade. Gincanas, vitrines de conhecimento, peças de teatro, jogos, são alternativas mais interessantes que palestras.

Desta forma, se faz necessário que estudos futuros sejam desenvolvidos nesta temática, visto que é um assunto importante para a saúde pública, que impacta diretamente no bem-estar dos adolescentes, e especialmente porque o conhecimento e divulgação desse tema precisa ser difundido e amplamente conhecido na literatura brasileira atualmente.

\section{Referências}

Almeida, R. A. A. S., Corrêa, R. D. G. C. F., Rolim, I. L. T. P., Hora, J. M. D., Linard, A. G., Coutinho, N. P. S., \& Oliveira, P. D. S. (2017). Conhecimento de adolescentes relacionados às doenças sexualmente transmissíveis e gravidez. Revista Brasileira de Enfermagem, 70, $1033-1039$.

Amoras, B. C., Campos, A. R., \& Beserra, E. P. (2015). Reflexões sobre vulnerabilidade dos adolescentes a infecções sexualmente transmissíveis. PRACS: Revista Eletrônica de Humanidades do Curso de Ciências Sociais da UNIFAP, 8(1), 163-171.

Andrade, P. S. P. D., Barros, K. R. D. S., Santos, J. P. D., Nascimento, E. F. D., \& Bacelar, P. A. A. (2021). Conhecimento de adolescentes e jovens sobre questões relacionadas ao sexo, em uma escola pública de Monte Alegre do Piauí-PI. Gerais: Revista Interinstitucional de Psicologia, 14(2), 1-23.

Botelho, L. L. R., de Almeida Cunha, C. C., \& Macedo, M. (2011). O método da revisão integrativa nos estudos organizacionais. Gestão e sociedade, 5(11), $121-136$.

Brasil, M. E., Cardoso, F. B., \& Silva, L. M. D. (2019). Conhecimento de escolares sobre infecções sexualmente transmissíveis e métodos contraceptivos. Revista de Enfermagem UFPE on line, 13, e242261.

Brasil. (2009). Ministério da Saúde. Secretaria de Atenção à Saúde. Departamento de Atenção Básica. Saúde na escola. Brasília.

Brasil. (2007). Presidência da República. Casa Civil. Subchefia para Assuntos Jurídicos. Decreto nº 6.286, de 5 de dezembro de 2007. Brasília: Casa Civil.

Brasil. (1999). República Federativa do Brasil. Ministério da Saúde. Comportamento Sexual da População Brasileira e Percepções sobre o HIV/AIDS. Relatório Final de Pesquisa. CNDST/HIV/AIDS. Brasília.

Câmara, S. C. (2012). Vulnerabilidades dos adolescentes à transmissão sexual do hiv/aids: uma análise no contexto do programa saúde na escola (Doctoral dissertation, dissertação]. Fortaleza: Universidade Estadual do Ceará.

da Silva, L. M., Cortes, E. A., do Espírito Santo, J. N., \& Cordeiro, B. C. (2017). Pesquisa-ação: promovendo educação em saúde com adolescentes sobre infecção sexualmente transmissível. Revista de Enfermagem UFPE on line, 11(9), 3642-3649.

de Andrade Ferreira, E., Alves, V. H., Pereira, A. V., Rodrigues, D. P., Paiva, E. D., \& dos Santos, I. M. M. (2018). Adolescentes no espaço escolar e o conhecimento a respeito da saúde sexual e reprodutiva. Cogitare Enfermagem, 23(2).

Ferreira, E. A., Alves, V. H., Pereira, A. V., Rodrigues, D. P., dos Santos, M. V., \& Gabrielloni, M. C. (2019). Sexualidade na percepção de adolescentes estudantes da rede pública de ensino: contribuição para o cuidado. Revista de Pesquisa: Cuidado é Fundamental, $1208-1212$.

Fonseca, F. F., Sena, R. K. R., Santos, R. L. A. D., Dias, O. V., \& Costa, S. D. M. (2013). As vulnerabilidades na infância e adolescência e as políticas públicas brasileiras de intervenção. Revista Paulista de Pediatria, 31, 258-264.

Fraenkel, E., Rasche, A. S., \& Santos, M. D. S. S. D. (2008). A enfermeira escolar e o seu objectivo. Esc. Anna Nery Rev. Enferm, 406-410.

Franco, M. D. S., Barreto, M. T. S., Carvalho, J. W. D., Silva, P. P. D., Moreira, W. C., Cavalcante, M. C., \& Lima, L. H. D. O. (2020). Educação em saúde sexual e reprodutiva do adolescente escolar. Rev. enferm. UFPE on line, 1-8.

Freire, A. K. S., de Melo, M. C. P., Vieira, M. P., Gomes, I. M., Gomes, J. L., de Sousa Ribamar, D., \& da Costa, M. M. (2017). Aspectos psicossociais da sexualidade na adolescência: diálogos e aprendizagem na escola. Semina: Ciências Biológicas e da Saúde, 38(1), 3-14.

Genz, N., Meincke, S. M. K., Carret, M. L. V., Corrêa, A. C. L., \& Alves, C. N. (2017). Doenças sexualmente transmissíveis: conhecimento e comportamento sexual de adolescentes. Texto \& Contexto-Enfermagem, 26.

Jones, D. E. (2010). Diálogos entre padres y adolescentes sobre sexualidad: discursos morales y médicos en la reproducción de las desigualdades de género. Interface-Comunicação, Saúde, Educação, 14, 171-182. 
Research, Society and Development, v. 11, n. 2, e3951125585, 2022

(CC BY 4.0) | ISSN 2525-3409 | DOI: http://dx.doi.org/10.33448/rsd-v11i2.25585

Lins, L. S., Silva, L. A. M., Santos, R. G., Morais, T. B. D., Beltrão, T. A., \& de Lima Castro, J. F. (2017). Análise do comportamento sexual de adolescentes. Revista Brasileira em Promoção da Saúde, 30(1).

Macedo, E. O. S., \& Conceição, M. I. G. (2015). Significados sobre la Adolescencia y la Salud entre los Participantes de un Grupo Educativo de Adolescentes. Psicologia: ciência e profissão, 35(4), 1059-1073.

Pinheiro, A. D. S., Silva, L. R. G. D., \& Tourinho, M. B. A. D. C. (2017). A estratégia saúde da família e a escola na educação sexual: uma perspectiva de intersetorialidade. Trabalho, Educação e Saúde, 15, 803-822.

Queiroz, A. A. F. L. N., de Sousa, Á. F. L., de Moura Feitosa, J. J., da Cunha Alves, R., Nery, I. S., \& Moura, M. E. B. (2016). Educação sexual para adolescentes por docentes de um centro de educação comunitária. Revista de Pesquisa: Cuidado é fundamental online, 8(4), 5120-5125.

Ribeiro, M. S. D. S., \& Ribeiro, C. V. (2015). Saúde e Prevenção nas Escolas (SPE): elementos para avaliação de projetos sociais em Juazeiro, Bahia, Brasil. Interface-Comunicação, Saúde, Educação, 19, 337-348.

Santos, S. C. D., Almeida, D. B. D., Oliveira, W. A. S. D., Alexandre, A. C. S., Lyra, F. M. P. D., \& Barbosa, V. F. B. (2017). A prevenção do vírus da imunodeficiência humana pela equipe de atenção primária voltada aos adolescentes. Rev. enferm. UFPE on line, 3050-3056.

Sehnem, G. D., Crespo, B. T. T., Lipinski, J. M., Ribeiro, A. C., Wilhelm, L. A., \& Arboit, J. (2019). Saúde sexual e reprodutiva dos adolescentes: percepções dos profissionais em enfermagem. Avances en Enfermería, 37(3), 343-352.

Silva, K. L. D., Dias, F. L. A., Maia, C. C., Pereira, D. C. R., Vieira, N. F. C., \& Pinheiro, P. N. D. C. (2010). A influência das crenças e valores culturais no comportamento sexual dos adolescentes do sexo masculino. Rev. enferm. UERJ, 247-252.

Silva, S. M. D. T. D., Vieira Ferreira, M. M. D. S., Amaral-Bastos, M. M., Monteiro, M. A. J., \& Couto, G. R. (2020). Diagnóstico do conhecimento dos adolescentes sobre sexualidade. Acta Paulista de Enfermagem, 33.

Souza, M. T. D., Silva, M. D. D., \& Carvalho, R. D. (2010). Revisão integrativa: o que é e como fazer. Einstein, 8, 102-106.

Souza, V. D., Pimenta, A. M., Caetano, L. C., Cardoso, J. S. R., Beinner, M. A., \& Villela, L. D. C. M. (2017). Conhecimentos, vivências e crenças no campo sexual: um estudo com alunos do ensino médio com perfis socioeconômicos diferenciados. Revista Mineira de Enfermagem, 21.

Vasconcelos, E. M. (2001). Redefinindo as práticas de saúde a partir de experiências de educação popular nos serviços de saúde. Interface-Comunicação, Saúde, Educação, 5, 121-126.

Veloso, D. L. C., Peres, V. C., Lopes, J. D. S. O. D. C., Salge, A. K. M., \& Guimarães, J. V. (2014). Anticoncepção de emergência: conhecimento e atitude de acadêmicos de enfermagem. Revista Gaúcha de Enfermagem, 35, 33-39.

Vieira, K. J., Barbosa, N. G., dos Santos Monteiro, J. C., de Almeida Dionízio, L., \& Gomes-Sponholz, F. A. (2021). Conhecimentos de adolescentes sobre métodos contraceptivos e infecções sexualmente transmissíveis. Revista Baiana de Enfermagem 35, .

Tronco, C. B., \& Dell'Aglio, D. D. (2012). Caracterização do comportamento sexual de adolescentes: iniciação sexual e gênero. Gerais: Revista Interinstitucional de Psicologia, 5(2), 254-269. 